\title{
Individualized treatments for the many
}

\author{
Broadening access to personalized medicines will require scalable and efficient manufacturing processes that help
} decrease production costs.

F or all the excitement around immunotherapies - and their undeniable potential for treating a wide range of diseases (including oncological, autoimmune and cardiovascular) - some patients do not benefit from them. For example, immune checkpoint inhibitors against many solid cancers, and chimeric antigen receptor (CAR)-T cells for treating some leukaemias and lymphomas, have proved to be efficacious and safe only for a fraction of patients (often less than half of those treated). This should not be surprising; tumours across disease types and across patients, as well as the patient's immune status, can be highly variable. To maximize the fraction of responders, therapeutic regimens can combine immunotherapies with cytotoxic anticancer drugs or radiation-based treatments. Also, patients can be selected according to their chances of responding to particular immunotherapies and combination therapies when suitable biomarkers exist, and closely monitored while under treatment so that any unexpected responses to the treatment and any adverse effects can be rapidly dealt with.

Exome sequencing and RNA sequencing, as well as proteomics pipelines, provide a pool of data that can be mined for mutations, neoantigens, altered biological pathways and other disease biomarkers. Whereas some strategies involve studying each patient individually for designing uniquely tailored therapies (such as neoantigen vaccines), large population studies such as the 'All of Us' project from the US National Institutes of Health aim to build a diverse database of biological and behavioural information from individuals across age groups, ethnic backgrounds, gender and health status. This wealth of data can then be used to tailor treatment types and regimens to individual patients or to subsets of patients with diseases of similar genetic background to maximize the chances of success.

The wealth of patient-specific data being produced will be increasingly capitalized by developments in gene-based vaccines, in the engineering of $\mathrm{T}$ cells and peptide vaccines, in combination immunotherapies, and in biomaterial-based drug-delivery solutions, as discussed by James Moon and colleagues in a Perspective in this issue. Patients have already benefited from personalized therapies (Fig. 1), most notably cancer vaccines that mount immune responses to unique antigens in the patient's tumour, and CAR-T cell therapies produced by modifying the patient's own $\mathrm{T}$ cells to awaken and boost immune responses to their tumours.

Adoptive cell therapies involve complex protocols that require cell products with high purity and potency. This is especially true for cancer therapies based on the insertion of CARs into patient-derived T cells. Suzie Pun and colleagues describe, in an Article in this issue, a 'traceless' method for the isolation of $\mathrm{CD}^{+} \mathrm{T}$ cells from peripheral blood mononuclear cells collected via leukapheresis from a patient's blood. The method relies on CD8-specific DNA aptamers instead of monoclonal antibodies (the latter remain attached to the cells during the purification process and may

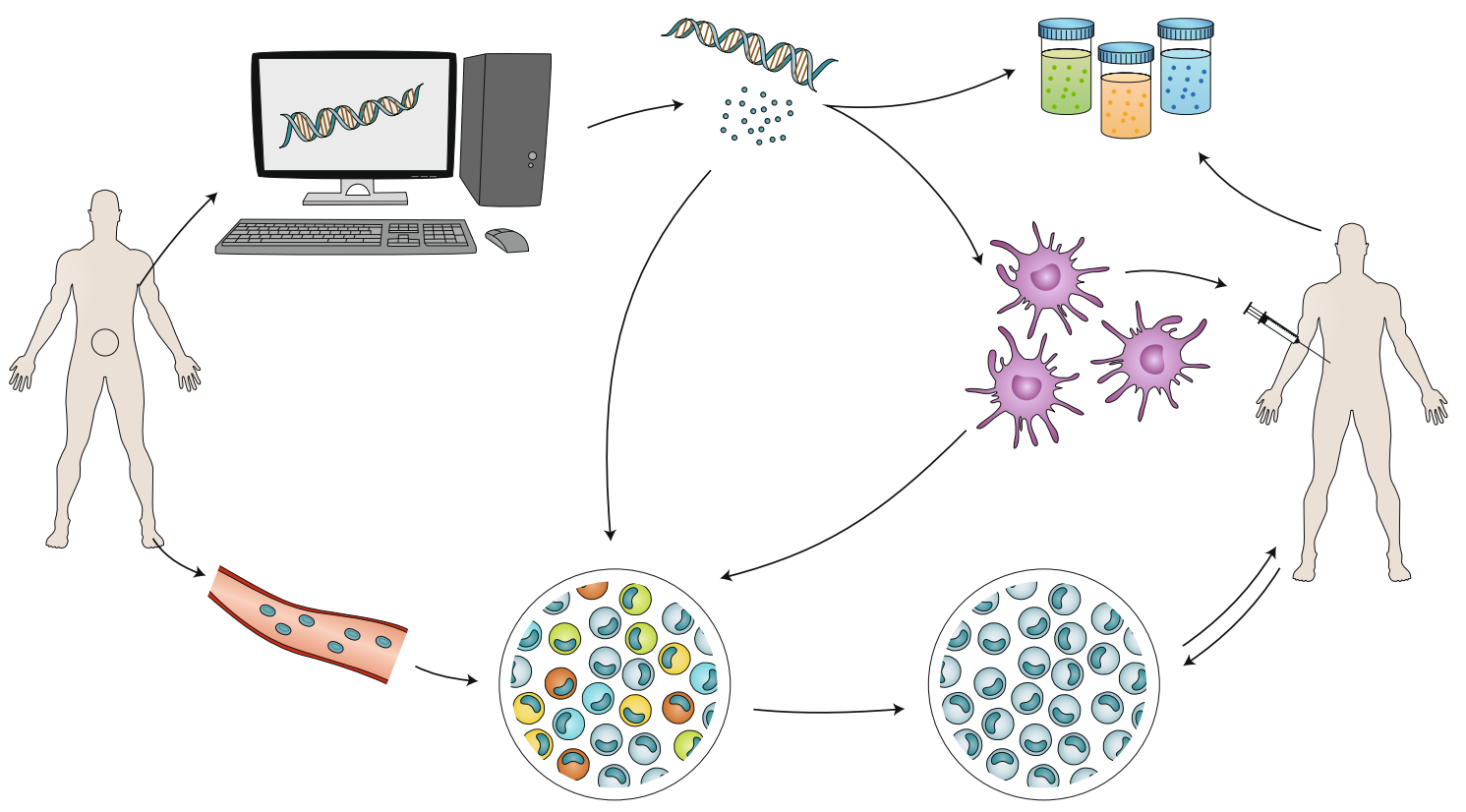

Fig. 1 | Patient-tailored immunotherapies. Neoantigens predicted from mutations identified in a patient's tumour sample can be leveraged to make

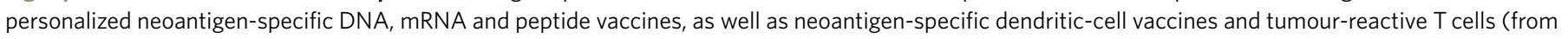
peripheral lymphocytes transduced with neoantigen-specific genes), which are then transferred back into the patient. Figure adapted from Scheetz, L. et al. https://doi.org/10.1038/s41551-019-0436-x (2019). 
trigger adverse immune responses against the modified cells after they are infused into the patient). The aptamers can be dislodged (by complementary oligonucleotides) from the CD8 receptor on the surface of the $\mathrm{T}$ cells after the isolation procedure. Because the approach is much less costly than the standard use of antibodies (or of fragment antigen-binding constructs), it could also contribute to making T-cell immunotherapies more accessible (CAR-T cell products and the latest gene therapies can currently cost hundreds of thousands of dollars per patient).

CRISPR-based genetic screens for the identification of genetic regulators of disease have extended the ability to probe patientspecific pathogenicities. However, a key limitation of genetic screens is the need to sort large numbers of cells. Although widely used fluorescence-activated cell-sorting methods can achieve remarkable purity, they lack the speed and scalability needed for high-throughput genetic screens. In this issue, Shana Kelley and colleagues show that a microfluidic chip for immunomagnetic cell sorting enables rapid and scalable lossof-function phenotypic CRISPR screens. In a proof-of-concept experiment, the researchers identified determinants of the surface presentation of CD47, a negative regulator of phagocytosis that is a target in cancer therapies. The enhanced performance of the chip could reduce the cost and time associated with sorting patient-derived cells, and may thus potentiate the interrogation of disease pathogenesis.

Beyond the efficient and cheaper isolation of patient-derived cell populations - one of the bottlenecks in the translation of cell therapies and in the investigation of patientspecific disease mechanisms - many other roadblocks can slow the translation of individualized therapies and limit access to personalized products. One big limitation is lengthy manufacture time: current protocols for the production of patientspecific neoantigen vaccines (including the removal and processing of tumour biopsies, the genome-sequencing of the samples, bioinformatics analyses for the identification and screening of suitable neoantigens, and the production of the vaccine) can take several months. The ex vivo expansion of patient-specific therapeutic T cells for adoptive transfer is also a long process.

Still, individualized treatments can partly rely on one-size-fits-all approaches; for example, the development of off-theshelf cell products engineered to avoid both acute and long-term immune responses in patients can form the basis of medicines that are then tailored to each patient. These would also help standardize some criteria (such as the physicochemical characteristics of candidate neoantigen peptides), as well as manufacturing protocols and processes (such as those for cell isolation and sorting) for the production of treatments suited to the individual characteristics of a patient's disease.

Published online: 7 October 2019 https://doi.org/10.1038/s41551-019-0468-2 